\title{
Diffusion of hydrogen in Pd assisted by inelastic ballistic hot electrons
}

\author{
M. Blanco-Rey, ${ }^{1,}$ M. Alducin, ${ }^{2}$ J.I. Juaristi, ${ }^{2,3}$ and P.L. de Andres ${ }^{1,4}$ \\ 1 Donostia International Physics Center, Universidad del País Vasco \\ UPV/EHU, Paseo Manuel de Lardizábal 4, 20018 Donostia-San Sebastián, Spain \\ ${ }^{2}$ Centro de Física de Materiales CFM/MPC (CSIC-UPV/EHU), \\ Paseo Manuel de Lardizábal 5, 20018 Donostia-San Sebastián, Spain \\ 3 Departamento de Física de Materiales, Facultad de Químicas \\ UPV/EHU, Apartado 1072, 20018 Donostia-San Sebastián, Spain \\ 4 Instituto de Ciencia de Materiales de Madrid, CSIC, Cantoblanco, 28049 Madrid, Spain
}

(Dated: March 8, 2022)

\begin{abstract}
Sykes et al. [Proc. Natl. Acad. Sci. 102, 17907 (2005)] have reported how electrons injected from a scanning tunneling microscope modify the diffusion rates of $\mathrm{H}$ buried beneath $\mathrm{Pd}(111)$. A key point in that experiment is the symmetry between positive and negative voltages for $\mathrm{H}$ extraction, which is difficult to explain in view of the large asymmetry in Pd between the electron and hole densities of states. Combining concepts from the theory of ballistic electron microscopy and electronphonon scattering we show that $\mathrm{H}$ diffusion is driven by the $s$-band electrons only, which explains the observed symmetry.
\end{abstract}

PACS numbers: 63.20.kd, 68.37.Ef, 71.20.Be

While the scanning tunneling microscope (STM) seems a natural tool to manipulate all sort of atoms and molecules adsorbed on surfaces [1], it is rather surprising to learn that tunneling currents can also be used to change diffusion rates of atoms deeply absorbed in metals. In an elegant experiment performed on $\mathrm{H}$ buried below the $\operatorname{Pd}(111)$ surface, Sykes et al. have unequivocally proved that this effect does exist [5]. Indeed, the STM has been used to investigate buried interfaces [6] or even individual molecules [7, but the pioneering experiment of Sykes et al. confirms the STM as a powerful nanometric technique to control diffusion and reaction processes at the atomic level not only on surfaces but also inside metals. The controlled insertion and extraction of $\mathrm{H}$ in $\mathrm{Pd}$ is an added interest of this particular experiment because of its possible applications in $\mathrm{H}$ storage 8 10 and in heterogenous catalysis. In this respect, $\mathrm{Pd}$ is a recurring catalyst in hydrocarbon synthesis reactions, particularly in $-\mathrm{C}=\mathrm{C}$ - bond breaking, and the reason behind such exceptional catalycity is precisely linked to the existence of weakly bound $\mathrm{H}$ at subsurface sites [11].

Still, the underlying mechanisms behind the selective $\mathrm{H}$ population of subsurface sites reported in [5] are not well understood. In particular, the observed bias voltage dependence is puzzling. Working at low temperature to prevent thermal diffusion $(T=4 \mathrm{~K})$, the STM is used as a local electron gun to inject small currents $\left(I_{t} \sim 1-150 \mathrm{pA}\right)$ in the vicinity of the Fermi energy $(V \sim \pm 1 \mathrm{~V})$. Inelastic excitation with the injected ballistic hot electrons promotes the diffusion of $\mathrm{H}$ from bulk $\mathrm{Pd}$ towards the surface even under low concentration conditions, thereby implying the contribution of large volumes of the metal where electric fields are efficiently screened out and cannot play a role. The distortions in the $\mathrm{Pd}-\mathrm{Pd}$ distances caused by subsurface $\mathrm{H}$ accumulation are then (a)

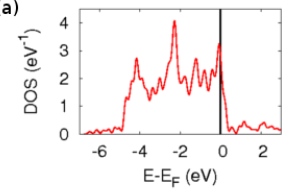

(c)

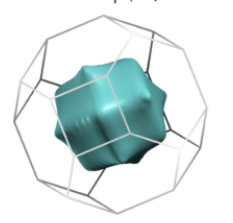

(b)

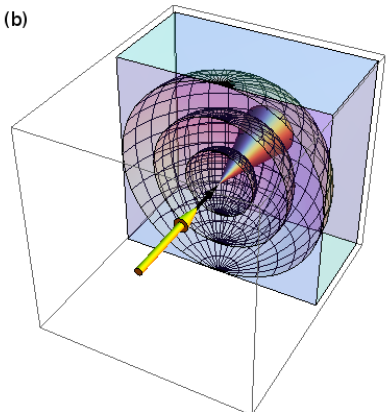

FIG. 1. (a) Calculated density of states for bulk Pd. (b) Sketch for injection of $s$-like (isotropic propagation), and $d$ like (narrowly focused) electrons on $\mathrm{Pd}(111)$. (c) Energy isosurface $\left(E_{F}+0.83 \mathrm{eV}\right)$ related to the $s$ band in Pd.

imaged with STM as protrusions or bright stripes. The brightness and width of the stripes increase with $I_{t}$ and $V$ magnitude. The intriguing finding is that the stripes are comparable in brightness for opposite $V$ signs, positive biases creating only slightly wider stripes. This polarity effect must have a non trivial explanation, since for $V>0$ and $V<0$ charge carriers are electrons and holes respectively, and the density of states above and below the Fermi level $E_{F}$ is very different in Pd [Fig. 1 (a)].

In the present letter, we examine the vibrational excitation of $\mathrm{H}$ in $\mathrm{Pd}$ caused by inelastic interaction with ballistic hot electrons using first order perturbation theory for the electron-phonon (e-ph) coupling. Hopping rates for $\mathrm{H}$ migration have been quantified as a function of the experimental $V$ and $I_{t}$. Making use of the theory developed to describe propagation in ballistic electron energy microscopy (BEEM) [12, we find that the observed symmetry between positive and negative $V$ follows when the different roles of $s$ and $d$-band electrons has been recog- 


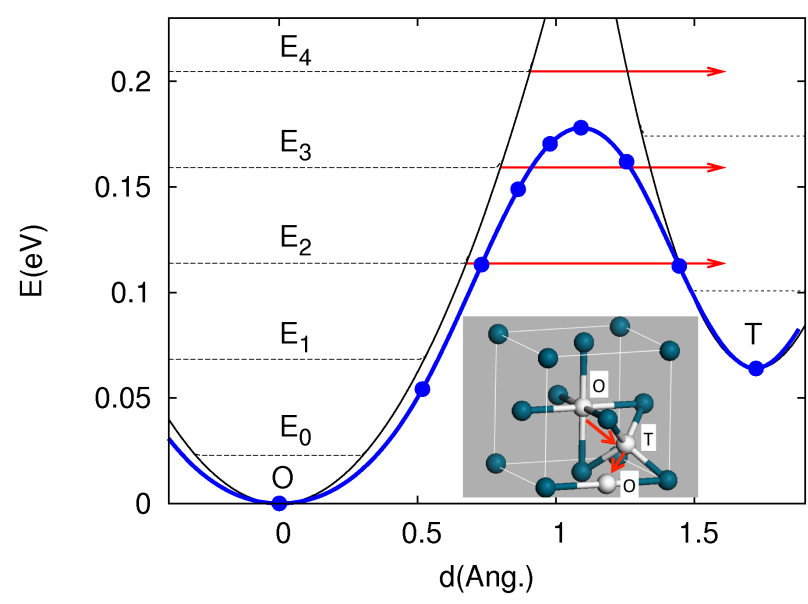

FIG. 2. (Color online) Profile of the computed energy barrier (blue thick line and circles) for diffusion from the octahedral $(\mathrm{O})$ to the tetrahedral site $(\mathrm{T})$. The approximated harmonic potential in $\mathrm{O}$ and $\mathrm{T}$ (black thin lines) and energy levels (dashed lines) are given for comparison. When $\mathrm{H}$ is excited up to a vibrational level $n \geq 2$, transmission by quantum tunneling becomes efficient and dominates in the intensity range of 1-150 pA. Inset: sketch of $\mathrm{O}$ and $\mathrm{T}$ sites (white) in the $\mathrm{Pd}$ fcc lattice (dark). Arrows mark the diffusion path from $\mathrm{O}$ to $\mathrm{T}$.

nized in the $\mathrm{H}$ vibrational excitation. It has been noticed that $d$-like electrons in bulk $\mathrm{Pd}$ propagate along narrow cones corresponding to a few selected $k$ points in the first Brillouin zone (1BZ) 13. Such a focusing effect happens typically when, with increasing energy, bands approaching the borders of the 1BZ change their curvature from convex to concave in order to touch the boundaries at right angles. While in a typical BEEM experiment focused beams are used to improve the resolution well below the surface, these electrons span a reduced substrate volume and have a small probability to interact with interstitial $\mathrm{H}$ in the low dilution regime. On the contrary, electrons propagating through $s$ channels span large volumes and are relevant for the present experiment because they are the only ones that can interact with many interstitial $\mathrm{H}$ [Fig. 1 (b) and (c)]. Interestingly our result shows that the inelastic excitation of $\mathrm{H}$ in $\mathrm{Pd}$ yields information about a component of the current that is hidden in a typical BEEM experiment, since it distributes over large spatial regions and does not contribute to nanometric resolution. Furthermore, we find that at $T=4 \mathrm{~K}$ the efficiency of the diffusion mechanism can only be understood by incorporating quantum tunneling of $\mathrm{H}$ near the top of the barrier. Similar phenomena have been in fact observed for heavier elements, e.g., deuterium in 14]. For this particular case, a larger mass would reduce the tunneling rate, change the frequencies of vibrational modes, and modify the rates in general. However, the relevant physics shall remain the same.
Ab-initio calculations have been performed by expanding wave functions on a plane-wave basis set using the Quantum Espresso package [15. This formalism yields accurate total energies, equilibrium geometries, electron band structures and transition states, as well as phonon frequencies, eigenvectors (in first order density functional perturbation theory), and e-ph coupling matrix elements. These are the ingredients for the inelastic model described below. The actual experiment is mimicked by using a periodic supercell with $\mathrm{Pd}_{16} \mathrm{H}$ stoichiometry. We have checked that such a supercell brings only small correlations between $\mathrm{H}$ atoms in consecutive cells. The theory level for the exchange and correlation functional is the generalized gradient approximation in the PerdewBurke-Ernzerhof formulation [16, and ion cores are described by ultrasoft pseudopotentials [17. In the construction of the plane wave basis sets we have used Monkhorst-Pack $k$-point meshes [18] and energy cut-offs for wave functions and charge densities of 28 and $180 \mathrm{Ry}$, respectively. The convergence threshold for total energy self-consistency is $10^{-10} \mathrm{Ry}$. The equilibrium lattice parameter obtained for fcc $\mathrm{Pd}$ is $3.98 \AA$. Using a $14 \times 14 \times 14$ Monkhorst-Pack mesh and the $\mathrm{Pd}_{16} \mathrm{H}$ cell, we determine that $\mathrm{H}$ is more stable at the octahedral site $(\mathrm{O})$ than at the tetrahedral site $(\mathrm{T})$ by $0.064 \mathrm{eV}$. The atomic positions of the $\mathrm{Pd}$ atoms surrounding the $\mathrm{H}$ atom have been relaxed with tolerances of $10^{-8} \mathrm{Ry}$ and $10^{-5} \mathrm{Ry} / \mathrm{a}$.u. in the energies and forces, respectively. The energy barrier from $\mathrm{O}$ to the metastable $\mathrm{T}$ has been found by the nudge elastic band method [19] to be $E_{b}=0.178 \mathrm{eV}$ (see Fig. 22]. This is in good agreement with the $0.23 \mathrm{eV}$ barrier found in electromigration experiments [20]. In the nudge elastic band calculation, nine images have been used and the final forces at the transition state are $\lesssim 5 \times 10^{-4} \mathrm{Ry} / \mathrm{a} . \mathrm{u}$.

Phonons have been calculated at the $\Gamma$ point using $10^{-12} \mathrm{Ry}$ as convergence criterion in the self-consistent loop. By restricting ourselves to $\vec{q}=0$ modes, we are neglecting correlations between $\mathrm{H}$ atoms in consecutive unit cells, which is exact in the dilute limit. Only the $\mathrm{H}$ atom and its six nearest neighboring $\mathrm{Pd}$ atoms are allowed to move in the phonon calculations. The three nondegenerate vibrational mode eigenvectors that yield a significant displacement of the $\mathrm{H}$ atom have energies of 17, 21 and $47 \mathrm{meV}$. The constraint on Pd atoms affects these values by only $\sim 5 \mathrm{meV}$.

In order to calculate $\mathrm{H}$ diffusion from $\mathrm{O}$ to $\mathrm{T}$ sites we approximate the $\mathrm{O}$ well by a harmonic potential truncated at an energy equal to $E_{b}$ 21 23. Figure 2 shows that the highest frequency mode $\hbar \omega=47 \mathrm{meV}$, in good agreement with the barrier profile, is the most likely to be involved in this process. Therefore, the $\mathrm{H}$ atom ought to be vibrationally excited up to approximately the fourth harmonic level to overcome the barrier and diffuse. The excited level populations obey a master equation that, in the limit of weak inelasticity, yields the following transfer 
rate from the $n$th vibrational excited level [22]:

$$
R_{n}=n \Gamma^{\uparrow}\left(\frac{\Gamma^{\uparrow}}{\Gamma^{\downarrow}}\right)^{n-1}
$$

where $\Gamma^{\uparrow}$ and $\Gamma^{\downarrow}$ are the vibrational excitation and deexcitation rates between the ground and first excited states. Hence, $R_{n}$ is obtained as the population of level $n-1$ times the probability to reach the $n$th vibrational excited state that is already above the top of the barrier.

Hot electrons injected from the STM tip into the substrate, or in the inverse direction, have energies in excess according to the bias voltage, $V$, and can excite vibrational quanta $(\hbar \omega)$ on the interstitial $\mathrm{H}$ via e-ph coupling. In first order time-dependent perturbation theory the transition rates for electron $(V>0)$ or hole $(V<0)$ decay can be obtained as:

$$
\begin{aligned}
\Gamma_{1}^{\uparrow, \downarrow}(\vec{q} ; \omega)= & \frac{2 \pi}{\hbar} \frac{1}{N_{\mathrm{Pd}}} \sum_{\epsilon_{i}, \epsilon_{j}} \sum_{\vec{k} \in 1 \mathrm{BZ}} \frac{w_{i \vec{k}}^{(s)}}{N_{\vec{k}}}\left|M_{i j}(\vec{k}, \vec{q} ; \omega)\right|^{2} \\
& \times \delta\left(\epsilon_{j}-\left(\epsilon_{i} \mp \hbar \omega\right)\right)
\end{aligned}
$$

where $\vec{k}$ and $\vec{q}$ are the electron and phonon wavevectors, respectively. The $\uparrow, \downarrow$ symbols account for $n \rightarrow n+1$ (vibrational excitation) and $n \rightarrow n-1$ (vibrational deexcitation) transitions, respectively. The initial and final electron band energies $\epsilon_{i, j}$ lie in the range $E_{F} \leq \epsilon_{i, j} \leq E_{F}+V$ for bias voltages $V>0$, and $E_{F}-|V| \leq \epsilon_{i, j} \leq E_{F}$ for $V<0$. The e-ph coupling matrix elements $M_{i j}(\vec{k}, \vec{q} ; \omega)$ are computed by Quantum Espresso [15]. Convergence on the sum over the 1BZ in Eq. 2 has been checked by using a sufficiently fine $k$-point grid [24]. $N_{\mathrm{Pd}}$ is the number of $\mathrm{Pd}$ atoms in the unit cell, a normalization factor ensuring that the rates do not depend on the cell size.

Now the crucial point is how to single out the injected electrons that are actually responsible for the $\mathrm{H}$ vibrational (de)excitation. With this purpose, we turn to the theory developed to describe propagation of hot electrons through a periodic lattice in BEEM [12. In its simplest version, BEEM theory uses a semiclassical Greens function due to Koster 25 to link the topography of the band-structure constant energy surface at $E_{F} \pm V$ with the spatial propagation of electrons in the substrate periodic lattice. For Pd, it predicts the formation of narrowly focused beams associated with the nearly flat $d$ bands, while electrons injected in $s$ bands propagate in a semispherical, $s$-wave-like form (see Fig. 1). For the experimental samples of diluted $\mathrm{H}$ in $\mathrm{Pd}$ we expect a behavior of the $s$ and $d$ bands similar to that found in bulk $\mathrm{Pd}$, since the low $\mathrm{H}$ concentration has a small impact on the $\mathrm{Pd}$ band structure. This has been seen in the $\mathrm{Pd}_{16} \mathrm{H}$ supercell, that already represents well the low-density regime in the experiment 26]. Therefore, electrons injected in the strongly focussed $d$ bands are not likely to encounter and, hence, to excite interstitial H. This mechanism can

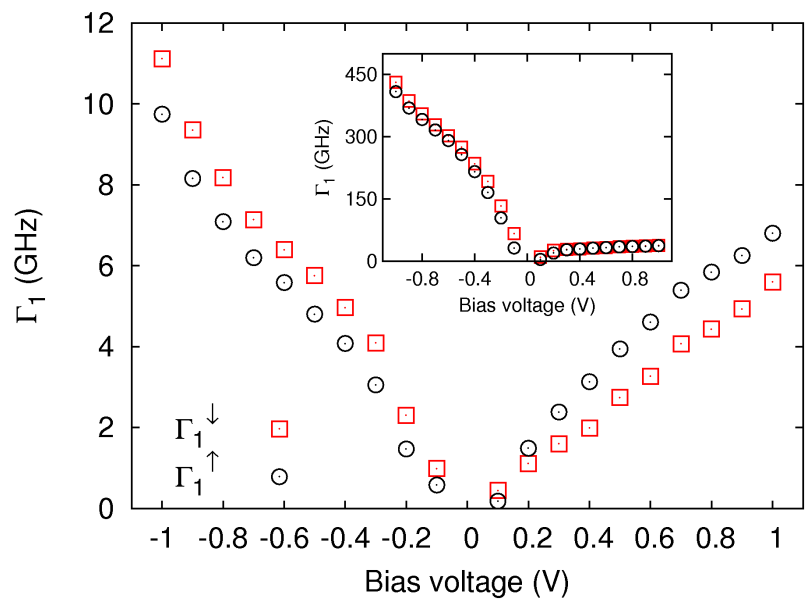

FIG. 3. Bias voltage dependence of the vibrational excitation $\left(\Gamma_{1}^{\uparrow}\right)$ and deexcitation $\left(\Gamma_{1}^{\downarrow}\right)$ rates as calculated from Eq. (2) when including the propagation correction through $w_{i \vec{k}}^{(s)}$ (see text). Inset: $\Gamma_{1}^{\uparrow, \downarrow}$ if both the $s$ and $d$ bands contribute in the injected electrons.

only be driven by the $s$ bands propagating in all directions in the crystal. To take into account this effect, we introduce in Eq. 2 weights, $w_{i \vec{k}}^{(s)}$, that have been obtained as the projected $s$-orbital percentage in the whole $\left|\psi_{i}(\vec{k})\right\rangle$ Kohn-Sham state. The calculated $\Gamma_{1}^{\uparrow, \downarrow}$ are plotted in Fig. 3. As seen in the inset, if both $s$ and $d$ states contributed fully, $\Gamma_{1}^{\uparrow, \downarrow}$ would map the Pd DOS and the large asymmetry around $E_{F}$ would cause differences in the rates of at least 2 orders of magnitude between positive and negative $V$. These differences are drastically reduced as soon as we realize that $\mathrm{H}$ excitation is mainly caused by nearly free ( $s$-like) electrons, that have a similar dispersion above and below $E_{F}$.

Rates in Eq. 2 are not yet the ones appearing in Eq. 1. The latter are the result of the net vibrational excitation caused by all the charge carriers in the incoming current, $I_{t}$. Therefore, we introduce the following linear model for $\Gamma^{\uparrow, \downarrow}$ :

$$
\Gamma^{\uparrow}=\frac{I_{t}}{e \Gamma_{0} \rho} \Gamma_{1}^{\uparrow}, \quad \Gamma^{\downarrow}=\Gamma_{0}+\frac{I_{t}}{e \Gamma_{0} \rho} \Gamma_{1}^{\downarrow}
$$

where $\Gamma_{0}$ is the deexcitation rate present at zero bias $\left[\Gamma_{0}\right.$ is calculated from Eq. 2 simply by removing the $w_{i \vec{k}}^{(s)}$ factors], and $\rho$ (see inset in Fig. 44 is the number of available one-electron states per Pd atom. By inserting this factor we are assuming that all the incoming carriers have an initial energy $\epsilon_{i}$ with equal probability $1 / \rho$, and also that the final state is always unoccupied. The latter is a fair assumption in the present low-intensity regime. Considering that $s$ carriers distribute evenly throughout the crystal and that the typical time that a $\mathrm{H}$ atom spends in a given excited vibrational state is $\sim 1 / \Gamma_{0} \sim 10^{-10} \mathrm{~s}$, the factor $\frac{I_{t}}{e \Gamma_{0}}$ represents the fraction 
of the current that (de)excites the $\mathrm{H}$ atom. The weak inelastic limit $\left(\Gamma^{\downarrow} \simeq \Gamma_{0} \gg \Gamma^{\uparrow}\right)$ holds well for the experimental intensities $I_{t}=1-150 \mathrm{pA}$. In particular, for $V= \pm 0.7 \mathrm{~V}$ and $I_{t}=150 \mathrm{pA}$ the effect of the injected hot electrons can be understood as if the interstitial $\mathrm{H}$ was effectively surrounded by a thermal bath at $T_{\text {eff }} \simeq 80 \mathrm{~K}$ according to the expression for a Botzmann distribution of level populations $k_{B} T_{\text {eff }}=\hbar \omega / \ln \left(\Gamma^{\downarrow} / \Gamma^{\uparrow}\right)[22$.

Quantum tunneling becomes important in the diffusion of nonmassive elements, like $\mathrm{H}$, specially at low temperatures. We introduce it in the model via a semiclassical (WKB) transmission rate through the barrier, $T\left(E_{n}\right)=e^{-2 \gamma\left(E_{n}\right)}$. To a very good approximation, a triangular-shaped barrier can be used instead of the upper part of the pathway in Fig. 2. In this case, $\gamma\left(E_{n}\right)=\frac{2}{3}\left[\frac{2 m_{H}}{\hbar^{2}}\left(E_{b}-E_{n}\right)\right]^{1 / 2} d\left(E_{n}\right)$, where $d\left(E_{n}\right)$ is the barrier width seen by $\mathrm{H}$ at the $n$-th vibrational level. We obtain $T\left(E_{2}\right)=5.1 \times 10^{-3}$ and $T\left(E_{3}\right)=0.24$. H transfer will occur as a result of climbing a $n$-step ladder and tunneling from this energy level with a combined transfer rate $R_{n} T\left(E_{n}\right)$. Since $R_{2} T\left(E_{2}\right)>R_{3} T\left(E_{3}\right) \gg R_{4}$, at the experimental currents of $1-150 \mathrm{pA}, n=2$ is the optimum level for the combined mechanism. Figure 4 shows $R_{2}$ for $I_{t}=100 \mathrm{pA}$. Values at different $I_{t}$ are estimated by noting that $R_{n}$ scales as $I_{t}^{n}$ in the weak inelastic limit. This scaling also shows that $\mathrm{H}$ transfer from the second and third vibrational states becomes comparable at larger currents $\left(I_{t}>1 \mathrm{nA}\right)$. Importantly, the similar rates obtained with positive and negative $V$ are consistent with the experimental insensitivity to the bias polarity. Furthermore, we observe in Fig. 4 a tendency to yield larger rates at $V \gtrsim 0.2 \mathrm{~V}$, consistent with the slightly wider stripes reported by Sykes et al. at $V>0$. This effect is a direct consequence of the distinct excitation dynamics of holes and electrons. More precisely, if the focusing of $d$ carriers had not been considered, the huge asymmetry in $\Gamma_{1}^{\uparrow, \downarrow}$ (see inset in Fig. 3 would have favored negative voltages over positive ones in $R_{n}$ (e. g. $>1$ order of magnitude in $R_{2}$ ).

In conclusion, we unravel the mechanism behind $\mathrm{H}$ diffusion in bulk $\mathrm{Pd}$ assisted by inelastic interaction with STM-injected hot electrons. Experiments reported for this system exhibit comparable $\mathrm{H}$ transfer for $V>0$ and $V<0$. This is a counter-intuitive result considering the strong difference between electron and hole band structures in $\mathrm{Pd}$. We find that both observations are reconciled by the fact that carriers from $\mathrm{Pd} d$ bands do not assist $\mathrm{H}$ diffusion, as their propagation is restricted to narrowly focused cones. The $\mathrm{H}$ vibrational excitation is only driven by the remaining $s$ carriers, more symmetrically distributed around $E_{F}$. Moreover, we find that at the experimental currents of 1-150 pA quantum tunneling is crucial to explain $\mathrm{H}$ diffusion (without it, the rates would be at least 2 orders of magnitude lower). Our model allows us to quantify the dependence of the diffu-

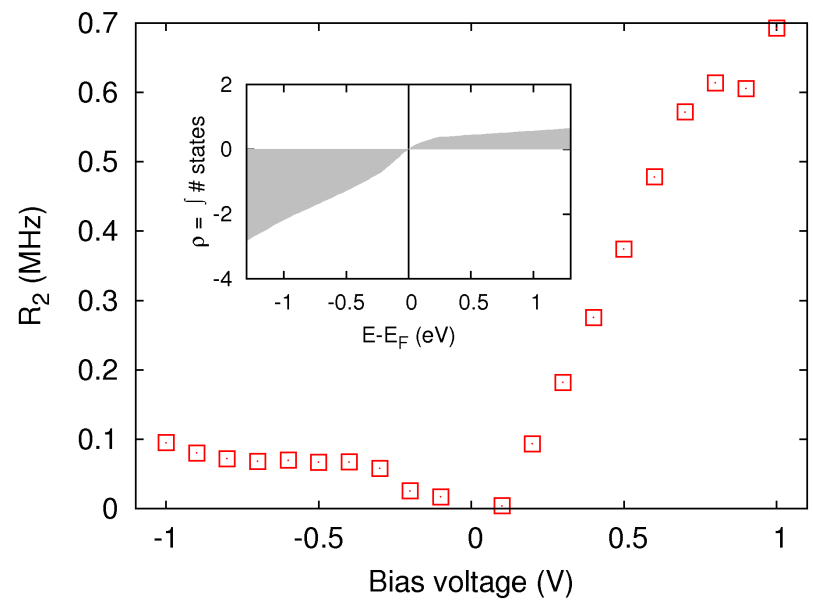

FIG. 4. (Color online) H transfer rate from the third excited vibrational state as a function of the bias voltage for $I_{t}=$ 100 pA. Inset: number of available initial states per Pd atom.

sion rates on the experimental $I_{t}$ and $V$ at low temperature. Since this mechanism is based in general concepts of solid-state theory i.e., the electronic band structure in a periodic potential, the propagation according to related Green functions, the e-ph interaction and tunneling of atoms at low temperatures, we believe our conclusions should apply to other transition metals.

We thank V. Chis and R. Díez Muiño for stimulating discussions. M.B.-R. acknowledges financial support from the Gipuzkoako Foru Aldundia and the European Commission Project No. FP7-PEOPLE-2010RG276921. This work is also supported by the Gobierno Vasco-UPV/EHU Project No. IT-366-07, and the Spanish MICINN Projects No. FIS2010-19609-C02-02 and No. MAT2011-26534. Computational resources were provided by the DIPC computing center.

* maria_blancorey@ehu.es

[1] J. A. Stroscio and D. M. Eigler, Science 254, 1319 (1991).

[2] B. C. Stipe, M. A. Rezaei, and W. Ho, Science 279, 1907 (1998).

[3] J. I. Pascual, N. Lorente, Z. Song, H. Conrad, and H.-P. Rust, Nature 423, 525 (2003).

[4] T. Komeda, Prog. Surf. Sci. 78, 41 (2005).

[5] E. C. H. Sykes, L. C. Fernandez-Torres, S. U. Nanayakkara, B. A. Mantooth, R. M. Nevin, and P. S. Weiss, Proc. Natl. Acad. Sci. 102, 17907 (2005).

[6] M. Prietsch, Phys. Rep. 253, 163 (1995).

[7] A. Bannani, C. Bobisch, and R. Möller, Science 315, 1824 (2007).

[8] L. Schlapbach and A. Züttel, Nature 414, 353 (2001).

[9] A. Pundt and R. Kirchheim, Ann. Rev. Mater. Res. 36, 555 (2006).

[10] J. C. Tremblay and P. Saalfrank, J. Chem. Phys. 131, 084716 (2009). 
[11] A. M. Doyle, S. K. Shaikhutdinov, S. D. Jackson, and H.-J. Freund, Angew. Chem. Int. Ed. 42, 5240 (2003).

[12] P. L. de Andres, F. J. Garcia-Vidal, K. Reuter, and F. Flores, Prog. Surf. Sci. 66, 3 (2001).

[13] F. Ladstadter, P. F. de Pablos, U. Hohenester, P. Puschnig, C. Ambrosch-Draxl, P. L. de Andres, F. J. Garcia-Vidal, and F. Flores, Phys. Rev. B 68, 085107 (2003).

[14] A. R. Kurland, P. Han, J. C. Thomas, A. N. Giordano, and P. S. Weiss, J. Phys. Chem. Lett. 1, 2288 (2010).

[15] P. Giannozzi, S. Baroni, N. Bonini, M. Calandra, R. Car, C. Cavazzoni, D. Ceresoli, G. L. Chiarotti, M. Cococcioni, I. Dabo, A. D. Corso, S. de Gironcoli, S. Fabris, G. Fratesi, R. Gebauer, U. Gerstmann, C. Gougoussis, A. Kokalj, M. Lazzeri, L. Martin-Samos, N. Marzari, F. Mauri, R. Mazzarello, S. Paolini, A. Pasquarello, L. Paulatto, C. Sbraccia, S. Scandolo, G. Sclauzero, A. P. Seitsonen, A. Smogunov, P. Umari, and R. M. Wentzcovitch, J. Phys.: Condens. Matter 21, 395502 (2009).

[16] J. P. Perdew, K. Burke, and M. Ernzerhof, Phys. Rev. Lett. 77, 3865 (1996).

[17] D. Vanderbilt, Phys. Rev. B 41, 7892 (1990).

[18] H. J. Monkhorst and J. D. Pack, Phys. Rev. B 13, 5188 (1976).

[19] G. Henkelman, B. P. Uberuaga, and H. Jónsson, J. Chem. Phys. 113, 9901 (2000).
[20] R. Pietrzak, R. Szatanik, and M. Szuszkiewicz, J. Alloy. Compd. 282, 130 (1999).

[21] R. E. Walkup, D. M. Newns, and P. Avouris, Phys. Rev. B 48, 1858 (1993).

[22] S. Gao, M. Persson, and B. I. Lundqvist, Phys. Rev. B 55, 4825 (1997).

[23] B. C. Stipe, M. A. Rezaei, W. Ho, S. Gao, M. Persson, and B. I. Lundqvist, Phys. Rev. Lett. 78, 4410 (1997).

[24] Summation in Eq. 2 collects contributions from small regions on the 1BZ; to perform the summation accurately the $M_{i j}(\vec{k}, 0 ; \omega)$ matrix elements are interpolated on a three times finer $k$-point grid than the original $14 \times 14 \times 14$ mesh. Errors in $\Gamma_{1}$ brought by $1 \mathrm{BZ}$ sampling effects are hence reduced to $\lesssim 0.7 \mathrm{GHz}$. The Dirac $\delta$ function is numerically calculated as a smearing Lorentzian function with half-width $s \ll \hbar \omega$. The choice $s=6 \mathrm{meV}$ yields errors $\lesssim 0.3 \mathrm{GHz}$ in $\Gamma_{1}$.

[25] G. F. Koster, Phys. Rev. 95, 1436 (1954).

[26] The bottom of the $d$ band is weakly contributed by H$\mathrm{Pd}$ hybridization and the states lying within $1 \mathrm{eV}$ above and below $E_{F}$ are unaffected by the inclusion of $H$. The $d$ band upper edge spills $\sim 0.2 \mathrm{eV}$ above $E_{F}$, and bands lying at higher energies correspond to strongly dispersive $s$-like states. The $\mathrm{H}$ states lie approximately $2-3 \mathrm{eV}$ below the bottom of the conduction band. 